\title{
ORIGINAL
}

\section{Determinants of time to death in hospital in critically ill patients around the world}

\author{
Ignacio Martin-Loeches ${ }^{1}$, Richard G. Wunderink², Rahul Nanchal ${ }^{3}$, Jean Yves Lefrant ${ }^{4}$, Farhad Kapadia ${ }^{5}$, \\ Yasser Sakr ${ }^{6}$ and Jean-Louis Vincent ${ }^{7 *}$, on behalf of the ICON Investigators
}

() 2016 Springer-Verlag Berlin Heidelberg and ESICM

\begin{abstract}
Purpose: To investigate which factors influence time to death in hospital in critically ill patients worldwide, including the possible impact of gross national income (GNI).

Methods: This was a pre-defined post hoc analysis of the Intensive Care Over Nations (ICON) database, which included 10,069 patients. For this sub-analysis, we included only the 206260 -day in-hospital non-survivors (22.3\%) among the 9258 patients with available hospital mortality and length-of-stay data. We categorized these non-survivors into three groups according to the time of death after ICU admission: early ( $<5$ days), intermediate ( $6-28$ days) or late ( $>28$ days).

Results: Time to death in hospital was early in 1068 of the 2062 non-survivors (52\%), intermediate in 808 (39\%), and late in $186(9 \%)$. Patients who died early had higher severity scores and were more likely to require mechanical ventilation on ICU admission, whereas those who died late were more likely to be older and to have had infection on ICU admission or during the ICU stay. Multilevel analysis indicated a stepwise increase in the risk of late or intermediate deaths according to increasing GNI. Patients admitted to ICUs in countries with high or upper-middle GNI were more likely to die late than those admitted to countries with low/lower-middle GNI [odds ratio ( $95 \%$ confidence interval) 4.78 (1.94-11.76), $p<0.001$, and $1.64(1.10-2.45), p=0.02$, respectively].
\end{abstract}

Conclusions: Duration of hospital stay prior to death in critically ill patients is longer in older patients, surgical patients, and patients with infection. GNI is a major determinant of time to death in hospital in these patients. These observations may have important organizational and ethical implications.

Keywords: Hospital mortality, Intensive care unit, Gross national income, Infection

*Correspondence: jlvincent@intensive.org

${ }^{7}$ Department of Intensive Care, Erasme Hospital, Université Libre de Bruxelles, Route de Lennik 808, 1070 Brussels, Belgium

Full author information is available at the end of the article

Take-home message: Duration of hospital stay prior to death in critically ill patients is longer in older patients, surgical patients, and patients with infection. Gross national income is a major determinant of time to death in these patients.

\section{Introduction}

Mortality rates in modern multidisciplinary intensive care units (ICUs) usually average 10-25 \% [1, 2]. Many of the deaths occur soon after admission due to the high severity of acute illness, however a substantial number of patients remain in the ICU for longer periods and eventually develop complications leading to late mortality [3]. In most of these latter cases, death is secondary to multiorgan failure and is anticipated, often leading to the decision to withdraw or limit life support; unexpected deaths rarely occur in the ICU [4]. However, the 
decision-making process in end-of-life situations is complex and requires the consideration of multiple factors, including severity of illness, physiological reserve, age, comorbidities, prior quality of life, and patient, family, and physician preferences.

Optimization of healthcare resources is a major concern worldwide, and in some countries efforts to save lives may be severely impaired due to the lack of resources. Financial incentives inherent in hospital payment methods may influence hospital lengths of stay (LOS). Maintaining the quality of care while lowering the associated costs is a hard sought-after goal of healthcare providers [5]. Two- to threefold differences in ICU riskadjusted mortality have been reported between centers, irrespective of the model that is used to adjust for severity of illness [6], and similar variations have been seen in risk-adjusted ICU LOS [7]. However, whether the level of healthcare resources in different regions of the globe influence the time to death due to critical illness is not clear.

The aim of the study reported here was to explore the independent factors associated with time to death in hospital in critically ill patients included in a large international database-the Intensive Care Over Nations (ICON) audit-with a special emphasis on the possible impact of the gross national income (GNI). We hypothesized that non-survivors in high-income countries may have longer LOS because sophisticated life support is more readily available and financial resources are sufficient to sustain prolonged organ support and allow for delays in end-of-life decision-making.

\section{Methods}

This is a pre-defined post hoc analysis of data from the ICON audit, which was conducted to better understand ICU patient demographics and ICU organization around the world. Full details on the methodology of this audit and definitions have been published elsewhere [8]. Participation was entirely voluntary, with no financial incentive. The participating institutions [Electronic Supplementary Material (ESM) Appendix 1] obtained ethical approval from their individual ethical review boards.

Each center prospectively collected data on all adult ( $>16$ years) patients admitted to their ICU between 8 May and 18 May 2012, except for those patients who stayed in the ICU for $<24 \mathrm{~h}$ for routine postoperative surveillance. Data were collected daily for a maximum of 28 days in the ICU. Data on hospital mortality were captured for up to 60 days.

Case report forms were electronically provided to the investigators using a secure internet-based website. Data on demographics and comorbid diseases were collected on admission. In this study the term surgical admissions refer to patients who had had surgery in the 4 weeks preceding admission to the ICU. Clinical and laboratory data for Simplified Acute Physiology Score II (SAPS) II [9] and Acute Physiologic Assessment and Chronic Health Evaluation (APACHE) II [10] scores were reported as the worst values within $24 \mathrm{~h}$ after admission. Microbiological data and clinical infections were reported daily. A daily evaluation of organ function according to the Sequential Organ Failure Assessment (SOFA) score was performed [11]; organ failure was defined as a SOFA score $>2$ for the organ in question.

Infection was defined according to the criteria of the International Sepsis Forum [12]. Sepsis was defined as the presence of infection with the concomitant occurrence of at least one acute organ failure attributed to infection. Septic shock was defined as sepsis associated with cardiovascular failure. Organ failure was judged not to be related to sepsis if it was already present $24 \mathrm{~h}$ before the onset of infection. ICU-acquired infection was defined as infection identified at least $48 \mathrm{~h}$ after ICU admission.

For the purposes of the sub-study, we included only non-surviving patients among those for whom hospital mortality and LOS data were available. Patients who remained alive in the hospital at day 60 were considered to be survivors for this analysis. We categorized hospital non-survivors into three groups according to the time to death after ICU admission in hospital: early ( $\leq 5$ days), intermediate (6-28 days), or late ( $\geq 28$ days) death. The cutoff for early mortality was chosen based on the median time of mortality in our population (5 days). The majority of published studies in critically ill patients use 28-day mortality as a primary outcome; therefore, we also used this cutoff to define late mortality.

For the purpose of this study, the 82 participating countries were classified into three income groups (low/lower middle, upper middle, and high) using the World Bank Atlas [13] classification of countries from 2011.

\section{Statistical analysis}

Data are shown as means with standard deviation, medians and interquartile ranges, or numbers and percentages. For continuous variables, the assumption of normality was checked by inspection of residual and normal plots. Differences between groups in terms of distribution of variables were assessed using analysis of variance, Chi-square test, or Fisher's exact test, as appropriate.

To determine the relative risk of being in the intermediate or late in-hospital mortality groups, we performed a multivariable analysis using a multilevel multinomial logistic model with three levels: patient, hospital, and country (for full details, see ESM). The dependent 
variable was time to death in hospital categorized into early (reference category), intermediate, and late. The explanatory variables considered in the model were:

- Patient-level factors: age, sex, SAPS II score, type of admission, source of admission, treatment with mechanical ventilation or renal replacement therapy (RRT) at any time during the ICU stay, comorbidities, presence of infection at any time during the ICU stay, and a decision to withhold/withdraw life-sustaining support at any time during the ICU stay.

- Hospital-level factors: type of hospital, ICU specialty, total number of ICU patients in 2011, number of staffed ICU beds.

- Country-level factors: GNI.

The results of fixed effects (measures of association) are given as odds ratios with their $95 \%$ confidence intervals. Random effects (measures of variation) measures included the variance (var). Data were analyzed using SPSS ${ }^{\circledR}$ Statistics software, version 22 for Windows (IBM Corp., Armonk, NY) and R software, version 2.0.1 (CRAN project; $\mathrm{R}$ Foundation for Statistical Computing, Vienna, Austria). All reported $p$ values are two-sided, and a $p$ value of $<0.05$ was considered to be statistically significant.

\section{Results}

We studied the 2062 non-survivors $(22.3 \%)$ among the 9258 patients for whom outcome and length of stay data were available. The median hospital LOS for these non-survivors was 5 (IQR 2-13) days after ICU admission. The demographic data of these patients are shown in Table 1 and the characteristics of each participating center are given in ESM Table e1. The mean patient age was 65 years, and mean SAPS II score was 57 points. An infection was present on ICU admission in 731 (35.5 \%) of the non-survivors, and more than half of all non-survivors $(1076,52.2 \%)$ had an infection at some point during the ICU stay (Table 2). Mechanical ventilation was already being administered to 1411 (68\%) of the nonsurvivors at ICU admission and to 1651 (80 \%) at some point during the ICU stay (Table 2).

Of the 2062 non-survivors, 1068 (52\%) died within the first 5 days in the ICU, 808 (39\%) died after 6-28 days, and $186(9 \%)$ died after $>28$ days. Patients who died early had higher severity scores (SAPS II and admission SOFA scores) than those with intermediate or late time to death. Patients who died early were more likely to be receiving mechanical ventilation on admission than those with intermediate or late time to death, but they were less likely to receive RRT during the ICU stay. Patients who died early more often had neurological, cardiovascular, respiratory and renal failures on ICU admission and neurological failure at any time during the ICU stay than those with intermediate or late time to death. Patients with intermediate or late time to death more often had coagulation or hepatic failure during the ICU stay than patients with early time to death (Table 2). A history of non-hematological cancer or corticosteroid administration prior to ICU admission was more common in patients with intermediate or late time to death, as was a diagnosis of infection at any time during the ICU stay.

The proportion of patients with a late time to death was higher in countries with a higher GNI than in those with a lower GNI [10.4 \% (high GNI) vs $7.7 \%$ (intermediate $\mathrm{GNI}$ ) vs $3.7 \%$ (low/low middle GNI); $p=0.001$ ]. The pattern was similar for patients with intermediate time to death ( 40.5 vs 38.7 vs 31.9 , respectively; $p=0.04$ ) (Table 2). Patients from high GNI countries with early time to death had higher SAPS II scores on ICU admission than patients from ICUs in low or upper middle GNI countries; patients admitted to ICUs in countries with high and upper middle GNI had higher SAPS II scores on ICU admission than patients from ICUs in low GNI countries; there were no differences in severity scores according to GNI in patients with late time to death (EMS Table e2).

\section{Associations with time to death in hospital}

The influence of patient, center, and country level characteristics on the time to death in hospital, with early mortality as the reference category, is presented in ESM Table e3. After controlling for patient and hospital factors and GNI (country factor), between-hospital variations remained significant for late time to death ( $v a r=0.56$, $p=0.03$ ); between-country variations in time to death were no longer present. Lower SAPS II scores and the presence of infection were independently associated with a higher risk of intermediate and late death. Presence of a decision to withhold/withdraw a life-sustaining measure was associated with a lower risk of intermediate or late death.

There was a stepwise increase in the risk of late death with increasing GNI (Fig. 1; ESM Table e3). Admission to an ICU in a country with a high or upper middle GNI was independently associated with a greater risk of late death than admission to a country with a low/lower middle GNI. Admission to an ICU with a high GNI was also independently associated with a greater risk of intermediate death compared to admission to a country with a low/lower middle GNI.

\section{Discussion}

To our knowledge, this is the first study to analyze the time to death in hospital for non-surviving patients 
Table 1 Differences in patient demographic variables upon intensive care unit admission according to time to death in hospital

\begin{tabular}{|c|c|c|c|c|c|}
\hline \multirow[t]{2}{*}{ Variables } & \multirow{2}{*}{$\begin{array}{l}\text { Total study cohort } \\
(n=2062)\end{array}$} & \multicolumn{3}{|c|}{ Time to death in hospital non-survivors ${ }^{a}$} & \multirow[t]{2}{*}{$p$ value } \\
\hline & & $\begin{array}{l}\text { Early deaths } \\
(n=1068)\end{array}$ & $\begin{array}{l}\text { Intermediate deaths } \\
(n=808)\end{array}$ & $\begin{array}{l}\text { Late deaths } \\
(n=186)\end{array}$ & \\
\hline $\operatorname{Age}^{\mathrm{b}}($ mean) & $65.2 \pm 16.7$ & $64.3 \pm 17.3$ & $66.2 \pm 16.0$ & $65.7 \pm 15.9$ & 0.05 \\
\hline SAPS II score (mean) & $56.6 \pm 19.3$ & $61.6 \pm 20.4$ & $51.5 \pm 16.4$ & $50.3 \pm 16.1$ & $<0.001$ \\
\hline SOFA score at admission (mean) & $9.3 \pm 4.7$ & $10.2 \pm 4.8$ & $8.4 \pm 4.3$ & $8.2 \pm 4.2$ & $<0.001$ \\
\hline Male ${ }^{b}$ & $1203(59.1)$ & $611(57.9)$ & $469(59.1)$ & $123(66.5)$ & 0.09 \\
\hline Type of admission to ICU ${ }^{b}$ & & & & & $<0.001$ \\
\hline Surgical & $511(26.0)$ & $222(21.9)$ & $221(28.4)$ & $68(38.4)$ & \\
\hline Medical & $1343(68.2)$ & $716(70.8)$ & $523(67.1)$ & $104(58.8)$ & \\
\hline Trauma & $97(4.9)$ & $62(6.1)$ & $30(3.9)$ & $5(2.8)$ & \\
\hline Other & $17(.9)$ & $12(1.2)$ & $5(.6)$ & $0(.0)$ & \\
\hline \multicolumn{6}{|l|}{ Comorbidities } \\
\hline COPD & $306(14.8)$ & $141(13.2)$ & $134(16.6)$ & $31(16.7)$ & 0.1 \\
\hline $\begin{array}{l}\text { Cancer (solid, } \\
\text { non-metastatic) }\end{array}$ & $265(12.9)$ & $118(11.0)$ & $121(15.0)$ & $26(14.0)$ & 0.04 \\
\hline Metastatic cancer & $113(5.5)$ & $50(4.7)$ & $50(6.2)$ & $13(7.0)$ & 0.23 \\
\hline Hematologic cancer & $96(4.7)$ & $49(4.6)$ & $41(5.1)$ & $6(3.2)$ & 0.55 \\
\hline $\begin{array}{l}\text { Diabetes mellitus (insulin- } \\
\text { dependent) }\end{array}$ & $214(10.4)$ & $103(9.6)$ & $89(11.0)$ & $22(11.8)$ & 0.5 \\
\hline Heart failure (NYHA III/IV) & $303(14.7)$ & $167(15.6)$ & $115(14.2)$ & $21(11.3)$ & 0.27 \\
\hline Chronic renal failure & $270(13.1)$ & $136(12.7)$ & $111(13.7)$ & $23(12.4)$ & 0.78 \\
\hline $\begin{array}{l}\text { Human immunodeficiency } \\
\text { virus infection }\end{array}$ & $25(1.2)$ & $14(1.3)$ & $8(1.0)$ & $3(1.6)$ & 0.72 \\
\hline Cirrhosis & $132(6.4)$ & $71(6.6)$ & $52(6.4)$ & $9(4.8)$ & 0.65 \\
\hline Immunosuppression & $112(5.4)$ & $48(4.5)$ & $53(6.6)$ & $11(5.9)$ & 0.14 \\
\hline Corticosteroid therapy & $106(5.1)$ & $47(4.4)$ & $42(5.2)$ & $17(9.1)$ & 0.03 \\
\hline Chemotherapy & $84(4.1)$ & $41(3.8)$ & $37(4.6)$ & $6(3.2)$ & 0.6 \\
\hline Number of comorbidities & & & & & 0.15 \\
\hline None & $864(41.9)$ & $472(44.2)$ & $318(39.4)$ & $74(39.8)$ & \\
\hline 1 & $628(30.5)$ & $323(30.2)$ & $245(30.3)$ & $60(32.3)$ & \\
\hline$>2$ & $570(27.6)$ & $273(25.6)$ & $245(30.3)$ & $52(28.0)$ & \\
\hline
\end{tabular}

Data are presented as the mean \pm standard deviation (SD) or as the number of patients with the percentage in parenthesis, as appropriate

COPD chronic obstructive pulmonary disease, ICU intensive care unit, NYHA New York Heart Association, SAPS Simplified Acute Physiology Score, SOFA Sequential Organ Failure Assessment

a Hospital non-survivors were categorized into three groups according to the time to death after ICU admission in hospital: early ( $\leq 5$ days), intermediate (6-28 days), or late ( $\geq 28$ days) death

b Data on age, sex, and type of admission were missing on eight, 28 , and 94 patients, respectively

admitted to ICUs around the world. Our key new findings relate to the impact of GNI on time to death in hospital. In the initial ICON study [8], we showed a strong relationship between the risk of in-hospital death and the GNI and suggested that differences in ICU organization between centers may play an important role in determining risk of death. In the current study, we approached this issue differently. Although LOS has been defined as a possible quality measure in ICU survivors, the LOS prior to death for critically ill patients has not been widely explored. The GNI classification provided an opportunity to detect significant differences in the time to death in hospital in critically ill patients according to country income. The proportion of late deaths in hospital was significantly higher in patients admitted to ICUs in high-income countries than in those admitted to ICUs in low-income ones. The greater proportion of late deaths in countries with a high GNI may be related to longer stays due to the greater availability of sophisticated lifesustaining technology and equipment in these countries. Prolonged ICU stay is a surrogate for advanced morbidity or perioperative complications, and intensive resource 
Table 2 Organ failure, complications, and gross national income in non-survivors according to time to death in hospital

\begin{tabular}{|c|c|c|c|c|c|}
\hline \multirow[t]{2}{*}{ Variables } & \multirow{2}{*}{$\begin{array}{l}\text { Total study cohort } \\
(n=2062)\end{array}$} & \multicolumn{3}{|c|}{ Time to death in hospital non-survivors ${ }^{a}$} & \multirow[t]{2}{*}{$p$ value } \\
\hline & & $\begin{array}{l}\text { Early } \\
(n=1068)\end{array}$ & $\begin{array}{l}\text { Intermediate } \\
(n=808)\end{array}$ & $\begin{array}{l}\text { Late } \\
(n=186)\end{array}$ & \\
\hline $\begin{array}{l}\text { Withdraw/withhold decision at any } \\
\text { time during ICU stay }\end{array}$ & $820(39.8)$ & $465(43.5)$ & $320(39.6)$ & $35(18.8)$ & $<0.001$ \\
\hline Infection at admission & $731(35.5)$ & $334(31.3)$ & $311(38.5)$ & $86(46.2)$ & $<0.001$ \\
\hline ICU-acquired infection & $237(11.5)$ & $40(3.7)$ & $157(19.4)$ & $40(21.5)$ & $<0.001$ \\
\hline Infection at any time during ICU stay & $1076(52.2)$ & $409(38.3)$ & $534(66.1)$ & $133(71.5)$ & $<0.001$ \\
\hline Mechanical ventilation at admission & $1411(68.4)$ & $759(71.1)$ & $526(65.1)$ & $126(67.7)$ & 0.02 \\
\hline Mechanical ventilation at any time & $1651(80.1)$ & $858(80.3)$ & $651(80.6)$ & $142(76.3)$ & 0.41 \\
\hline RRT at admission & $199(9.7)$ & $113(10.6)$ & $65(8.0)$ & $21(11.3)$ & 0.13 \\
\hline RRT at any time & $478(23.2)$ & $202(18.9)$ & $222(27.5)$ & $54(29.0)$ & $<0.001$ \\
\hline \multicolumn{6}{|c|}{ Type of organ failure at admission (alone or in combination) } \\
\hline Cardiovascular & $976(47.3)$ & $576(53.9)$ & $330(40.8)$ & $70(37.6)$ & $<0.001$ \\
\hline Respiratory & $793(38.5)$ & $437(40.9)$ & $292(36.1)$ & $64(34.4)$ & 0.05 \\
\hline Coagulation & $219(10.6)$ & $120(11.2)$ & $81(10.0)$ & $18(9.7)$ & 0.64 \\
\hline Hepatic & $249(12.1)$ & $121(11.3)$ & $105(13.0)$ & $23(12.4)$ & 0.54 \\
\hline CNS & $934(45.3)$ & $589(55.1)$ & $288(35.6)$ & $57(30.6)$ & $<0.001$ \\
\hline Renal & $709(34.4)$ & $440(41.2)$ & $216(26.7)$ & $53(28.5)$ & $<0.001$ \\
\hline \multicolumn{6}{|c|}{ Type of organ failure at any time (alone or in combination) } \\
\hline Cardiovascular & $1346(65.3)$ & $705(66.0)$ & $534(66.1)$ & $107(57.5)$ & 0.07 \\
\hline Respiratory & $1207(58.5)$ & $590(55.2)$ & $510(63.1)$ & $107(57.5)$ & $<0.01$ \\
\hline Coagulation & $461(22.4)$ & $186(17.4)$ & $224(27.7)$ & $51(27.4)$ & $<0.001$ \\
\hline Hepatic & $548(26.6)$ & $203(19.0)$ & $265(32.8)$ & $80(43.0)$ & $<0.001$ \\
\hline CNS & $1249(60.6)$ & $705(66.0)$ & $454(56.2)$ & $90(48.4)$ & $<0.001$ \\
\hline Renal & $1368(66.3)$ & $713(66.8)$ & $525(65.0)$ & $130(69.9)$ & 0.4 \\
\hline GNI category ${ }^{b}$ & & & & & $<0.001$ \\
\hline Low/lower middle & $188(100)$ & $121(64.4)$ & $60(31.9)$ & $7(3.7)$ & \\
\hline Upper middle & $595(100)$ & $319(53.6)$ & $230(38.7)$ & $46(7.7)$ & \\
\hline High & $1279(100)$ & $628(49.1)$ & $518(40.5)$ & $133(10.4)$ & \\
\hline \multicolumn{6}{|l|}{ Deaths } \\
\hline In the ICU & $1557(75.5)$ & 989 (92.6) & $512(63.4)$ & $56(30.1)$ & $<0.001$ \\
\hline On the general floor & $505(24.5)$ & $79(7.4)$ & $296(36.6)$ & $130(69.9)$ & $<0.001$ \\
\hline
\end{tabular}

Data are presented as the number of patients with the percentage in parenthesis

CNS Central nervous system, GNI gross national income, RRT renal replacement therapy

a Hospital non-survivors were categorized into three groups according to the time to death after ICU admission in hospital: early ( $\leq 5$ days), intermediate (6-28 days), or late ( $\geq 28$ days) death

b Horizontal percentages. The 82 participating countries were classified into three income groups (low/lower middle, upper middle, and high) using the World Bank Atlas [13] classification of countries from 2011

use, including prolonged mechanical ventilation, may be required in these patients. A recent study in Australia and New Zealand analyzed patients with persistent critical illness and reported that such patients consumed more resources and had higher mortality rates than patients with acute critical illness [14]. In contrast, critical care facilities remain underdeveloped in resourcelimited countries, and prolonged organ support may not be practically feasible or financially viable. Importantly, we identified within-country interhospital variation in terms of the duration of stay in hospital before death, suggesting that differences in local ICU organization, including timing of end-of-life decisions, may also influence the time to death. Improved healthcare, preventive strategies, and technology on a worldwide basis, as well as analysis of the cost-effectiveness of these approaches, represent challenges for the future of intensive care and require further attention [15].

Patients who died late (after 28 days) were more likely to have been admitted to the ICU with an infection, and 


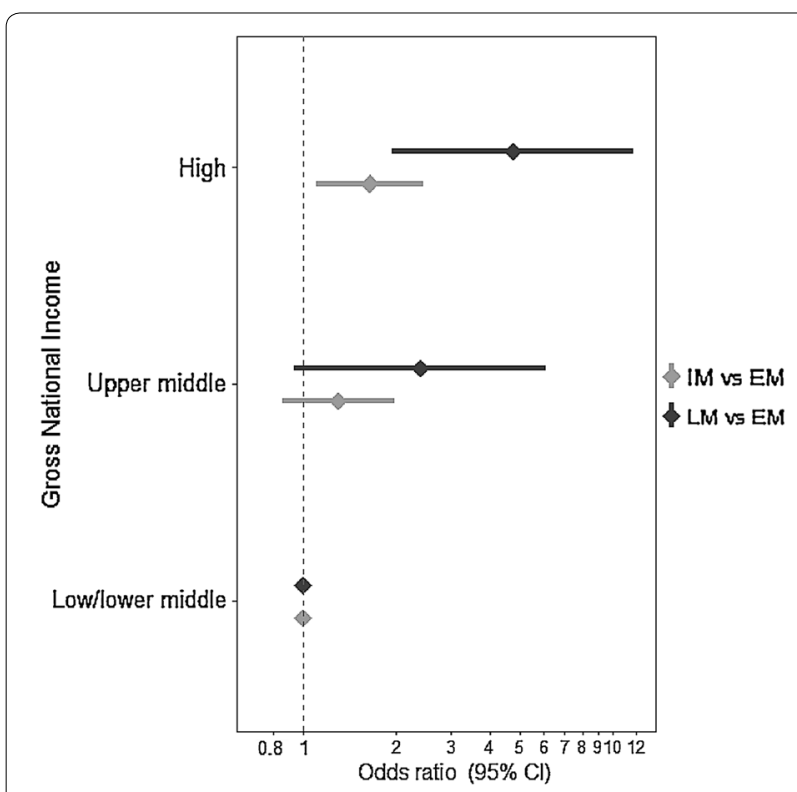

Fig. 1 Risk of intermediate or late death versus early death according to country gross national income (GNI). There is a stepwise increase in the risk of a longer time interval prior to death with increasing GNI. EM early mortality, IM intermediate mortality, LM late mortality

presence of infection during the ICU stay was independently associated with a higher risk of late death in hospital. One possible explanation for this association of infection with later death is that physicians continue to treat such patients in the hope that the infection may be cured with appropriate antibiotics and/or better source control, leading to prolonged ICU stays and later deaths. Alternatively, the relationship between presence of infection and longer time to death may also simply indicate that if a patient stays in hospital longer, he or she is at higher risk of a nosocomial infection.

In the multivariable analysis, older age was significantly associated with a greater risk of later time to death in hospital. Elderly patients are more likely to be frail and have diminished physiological reserve than younger patients [16]. This makes them more vulnerable to the adverse neuromuscular and cognitive sequelae of critical illness and potentially reduces their ability to recover sufficiently to leave the hospital despite having survived the initial insult. It is also possible that older patients are more vulnerable to nosocomial infections, which are acquired later in the ICU stay. ICU patients are increasingly elderly, and the relative contributions of age and comorbidities on late mortality are often difficult to determine. In the article reporting the initial results from the ICON audit, older age and severity of illness were strongly associated with mortality in hospital [8]. In the present analysis, patients from high income countries had higher disease severity on admission; however, these data did not impact on the final analysis when adjusted. A recent prospective study showed that patient characteristics at ICU discharge, including age, were the strongest predictors of later hospital death [17]. In patients with septic shock, Daviaud et al. [3] recently reported that age, malignancy, diabetes mellitus, no pathogen identification, and initial SOFA score were independently associated with ICU death within 3 days of ICU admission, whereas age, cirrhosis, no pathogen identification, and previous corticosteroid treatment were associated with ICU death at $>3$ days after ICU admission.

As expected, those who died early were more severely ill and had more severe organ failure, especially neurological, cardiovascular, respiratory, and renal, on ICU admission. Patients who died late more often developed hepatic dysfunction and coagulopathy. This finding is consistent with previous reports of failure of the hepatic and coagulation systems heralding a complicated clinical course, poor outcomes and higher mortality rates among critically ill patients [2]. Interestingly, the presence of different comorbid conditions was similar across the groups, apart from the presence of solid cancer and previous corticosteroid therapy, which were more prevalent in the cohort of patients with late deaths. In patients with septic shock, Daviaud et al. [3] also reported that previous corticosteroid administration was an independent predictor of late death.

In our study, surgical patients were more likely to die later, perhaps because in these patients major complications can occur late and decisions to forgo life support may be delayed. Additionally, mortality within 30 days of surgery is a global quality indicator and may influence the timing of end-of-life decision-making in these patients [18].

We acknowledge there are a number of limitations to our study. First, the registry was voluntary, and centers with higher academic resources may have been overrepresented. Similarly, the proportion of low income countries was less than that of high income countries; however the analyses performed, including those with fixed effects (varying within clusters), were conducted to minimize center and country bias. Second, data were collected for a single period of 10 days and so do not reflect any influence of seasonal variation. Third, comorbidities were coded as categorical variables, and no information on the level of severity of these conditions was available. Fourth, we defined LOS as starting from ICU admission and did not take into account pre-ICU hospital stays. Fifth, the study was focused on ICU management, so that information on complications occurring after hospital discharge and on the regular floor after ICU discharge was limited. Finally, we have no information on patient, 
physician, and family end-of-life preferences, culture, religion, or individual institutional or country characteristics, all factors that can play an important role in endof-life decision-making processes [19].

In summary, the duration of hospital stay prior to death in non-surviving critically ill patients is generally longer in older patients, in surgical patients, and in patients with infection. Later deaths in hospital are also more common in higher income countries. These observations may have important organizational and ethical implications.

\section{Electronic supplementary material}

The online version of this article (doi:10.1007/s00134-016-4479-0) contains supplementary material, which is available to authorized users.

\section{Author details \\ ${ }^{1}$ Corporacion Sanitaria Parc Taulí, CIBER Enfermedades Respiratorias, Parc Tauli University Institute, Sabadell, Spain. ${ }^{2}$ Northwestern University Feinberg School of Medicine, Chicago, IL, USA. ${ }^{3}$ Department of Medicine, Medical Col- lege of Wisconsin, Milwaukee, WI, USA. ${ }^{4}$ Service FOREVA, Division Anesthésie Réanimation Douleur Urgence, CHU Nîmes, Nîmes, France. ${ }^{5}$ Department of Critical Care, Hinduja Hospital, Mumbai, India. ${ }^{6}$ Department of Intensive Care, Universitätsklinikum Jena, Jena, Germany. ${ }^{7}$ Department of Intensive Care, Erasme Hospital, Université Libre de Bruxelles, Route de Lennik 808, 1070 Brussels, Belgium.}

\section{Acknowledgments}

The authors would like to thank Hassane Njimi for his help with the statistical analyses.

\section{Compliance with ethical standards}

\section{Conflicts of interest}

The authors declare that they have no conflicts of interest to declare.

Received: 2 June 2016 Accepted: 29 July 2016

Published online: 12 August 2016

\section{References}

1. Brinkman S, Bakhshi-Raiez F, Abu-Hanna A, de Jonge E, de Keizer NF (2013) Determinants of mortality after hospital discharge in ICU patients: literature review and Dutch cohort study. Crit Care Med 41:1237-1251

2. Sakr Y, Lobo SM, Moreno RP, Gerlach H, Ranieri VM, Michalopoulos A, Vincent JL (2012) Patterns and early evolution of organ failure in the intensive care unit and their relation to outcome. Crit Care 16:R222

3. Daviaud F, Grimaldi D, Dechartres A, Charpentier J, Geri G, Marin N, Chiche JD, Cariou A, Mira JP, Pène F (2015) Timing and causes of death in septic shock. Ann Intensive Care 5:16
4. Mayr VD, Dunser MW, Greil V, Jochberger S, Luckner G, Ulmer H, Friesenecker BE, Takala J, Hasibeder WR (2006) Causes of death and determinants of outcome in critically ill patients. Crit Care 10:R154

5. Tsai TC, Jha AK (2014) Hospital consolidation, competition, and quality: is bigger necessarily better? JAMA 312:29-30

6. Shortell SM, Zimmerman JE, Gillies RR, Duffy J, Devers KJ, Rousseau DM, Knaus WA (1992) Continuously improving patient care: practical lessons and an assessment tool from the National ICU Study. QRB Qual Rev Bull 18:150-155

7. Sakr Y, Moreira CL, Rhodes A, Ferguson ND, Kleinpell R, Pickkers P, Kuiper MA, Lipman J, Vincent JL (2015) The impact of hospital and ICU organizational factors on outcome in critically ill patients: Results from the extended prevalence of infection in intensive care study. Crit Care Med 43:519-526

8. Vincent JL, Marshall JC, Namendys-Silva SA, Francois B, Martin-Loeches I, Lipman J, Reinhart K, Antonelli M, Pickkers P et al (2014) Assessment of the worldwide burden of critical illness: the intensive care over nations (ICON) audit. Lancet Respir Med 2:380-386

9. Le Gall JR, Lemeshow S, Saulnier F (1993) A new Simplified Acute Physiology Score (SAPS II) based on a European/North American multicenter study. JAMA 270:2957-2963

10. Knaus WA, Draper EA, Wagner DP, Zimmerman JE (1985) APACHE II: a severity of disease classification system. Crit Care Med 13:818-829

11. Vincent JL, Moreno R, Takala J, Willatts S, De Mendonca A, Bruining H, Reinhart CK, Suter PM, Thijs LG (1996) The SOFA (Sepsis-related Organ Failure Assessment) score to describe organ dysfunction/failure. On behalf of the Working Group on Sepsis-Related Problems of the European Society of Intensive Care Medicine. Intensive Care Med 22:707-710

12. Calandra T, Cohen J (2005) The international sepsis forum consensus conference on definitions of infection in the intensive care unit. Crit Care Med 33:1538-1548

13. The World Bank. GNI per capita, Atlas method (current US\$). Available at: http://data.worldbank.org/indicator/NY.GNP.PCAP.CD. Accessed Jan 2012

14. Iwashyna TJ, Hodgson CL, Pilcher D, Bailey M, van Lint A, Chavan S, Bellomo R (2016) Timing of onset and burden of persistent critical illness in Australia and New Zealand: a retrospective, population-based, observational study. Lancet Respir Med 4:566-573

15. Gomersall CD (2010) Critical care in the developing world - a challenge for us all. Crit Care 14:131

16. Bagshaw SM, Stelfox HT, McDermid RC, Rolfson DB, Tsuyuki RT, Baig N, Artiuch B, Ibrahim Q, Stollery DE et al (2014) Association between frailty and short- and long-term outcomes among critically ill patients: a multicentre prospective cohort study. CMAJ 186:E95-102

17. Santamaria JD, Duke GJ, Pilcher DV, Cooper DJ, Moran J, Bellomo R (2015) The timing of discharge from the intensive care unit and subsequent mortality. A prospective, multicenter study. Am J Respir Crit Care Med 191:1033-1039

18. Watters DA, Hollands MJ, Gruen RL, Maoate K, Perndt H, McDougall RJ, Morriss WW, Tangi V, Casey KM, McQueen KA (2015) Perioperative mortality rate (POMR): a global indicator of access to safe surgery and anaesthesia. World J Surg 39:856-864

19. Curtis JR, Vincent JL (2010) Ethics and end-of-life care for adults in the intensive care unit. Lancet 376:1347-1353 